University of South Carolina

Scholar Commons

$10-19-2015$

\title{
No Vacuum Cerenkov Radiation Losses in the Timelike Lorentz- Violating Chern-Simons Theory
}

Karl Schober

Brett David Altschul

altschul@mailbox.sc.edu

Follow this and additional works at: https://scholarcommons.sc.edu/phys_facpub

Part of the Physics Commons

Publication Info

Published in Physical Review D, Volume 92, Issue 12, 2015.

(C) 2015 American Physical Society

This Article is brought to you by the Physics and Astronomy, Department of at Scholar Commons. It has been accepted for inclusion in Faculty Publications by an authorized administrator of Scholar Commons. For more information, please contact digres@mailbox.sc.edu. 


\title{
No Vacuum Cerenkov Radiation Losses in the Timelike Lorentz-Violating Chern-Simons Theory
}

\author{
Karl Schober, Brett Altschul1 \\ Department of Physics and Astronomy \\ University of South Carolina \\ Columbia, SC 29208
}

\begin{abstract}
In a Lorentz- and CPT-violating modification of electrodynamics that includes a timelike Chern-Simons term, there are no energy losss through vacuum Cerenkov radiation. A charge moving with a constant velocity does not lose energy, because of an unusual cancellation. Higher frequency modes of the electromagnetic field carry away positive energy, but lower frequency modes carry away a compensating negative amount of energy.
\end{abstract}

\footnotetext{
${ }^{1}$ baltschu@physics.sc.edu
} 
Recent years have seen a growth in interest in the possibility that Lorentz and CPT symmetries might be violated at the most fundamental levels of physics. These symmetries are ordinarily considered basic building blocks for the laws of nature, but they might not hold in theories of quantum gravity. In fact, many of the theories that have been proposed as candidate theories of quantum gravity may have regimes with Lorentz symmetry violation. Conversely, if any violations of Lorentz or CPT symmetries are ever uncovered experimentally, that will be a discovery of paramount importance, opening up new windows on the structure of fundamental physics.

There are also other motivations for studying exotic symmetry-breaking theories. Even if Lorentz violation and CPT violation are not found to exist in nature, by studying theories with these kinds of symmetry breakings, we may gain new insights about the basic structure of quantum field theories. Such studies may be conducted using effective quantum field theory. There is an effective theory called the standard model extension (SME) that has been developed to deal with Lorentz and CPT violation in fairly general fashion. The action for the SME contains all operators that can be constructed using standard model fields [1, 2]. Much more general operators are possible in the SME than in the standard model itself, because the operators are not constrained by Lorentz invariance. While the full SME obviously contains an infinite number of possible operators, the minimal SME, which includes only local, gauge invariant, and superficially renormalizable operators, provides a tractable test theory for exploring the experimental implications of Lorentz and CPT violations.

This paper will explore a particularly interesting process that can occur in many Lorentz-violating modifications of quantum electrodynamics - vacuum Cerenkov radiation, in which a charged particle moving with constant velocity through the vacuum emits radiation. Despite all appearances to the contrary (and expectations based on the usual threshold condition for Cerenkov emission), charges in uniform motion in the theory we shall consider do not lose energy through Cerenkov radiation.

Although it is possible to have Lorentz violation in both the electromagnetic and matter sectors, we shall concentrate on one particular form of Lorentz violation that is purely electromagnetic. The term we shall consider - the timelike electromagnetic ChernSimons term - is one of the most interesting terms that appear in the SME action. This Chern-Simons term introduces a dimensional scale into pure electrodynamics. It leads to a screening of magnetostatic fields and differing dispersion relations for right- and left-handed electromagnetic waves. This kind of birefringence would have a distinctive experimental signature, which has been searched for and not seen, even for photons that have traversed cosmological distances [3, 4]. This leads to exceedingly strong experimental constraints on the coefficient of the Chern-Simons operator.

However, in spite of these tight empirical bounds, the Chern-Simons term remains extremely interesting; understanding the behavior of quantum electrodynamics with an added Chern-Simons term may reveal new insights about how quantum field theories may behave. For example, the Chern-Simons term in the SME Lagrange density is not 
gauge invariant; however, since the term changes by a total derivative under a gauge transformation, the integrated action associated with the Chern-Simons term is gauge invariant. This subtlety makes determining the radiative corrections to the Chern-Simons term an extremely tricky problem, which led to some significant controversy [5, 6, 7, 8, 9, 10.

Naively, vacuum Cerenkov radiation looks like it should be allowed in the ChernSimons theory. Ordinary Cerenkov radiation only occurs in a medium, because in a Lorentz-invariant vacuum, a process like $e^{-} \rightarrow e^{-}+\gamma$ is disallowed by energy-momentum conservation. However, it is common in Lorentz-violating theories for processes that are normally kinematically forbidden to become allowed. The naive condition for Cerenkov emission - that the speed of a moving charge exceeds the phase speed of light propagating in the same direction - can be readily satisfied in the Chern-Simons theory. However, we shall see that whether Cerenkov losses actually occur in this theory is a much more subtle question.

The question of vacuum Cerenkov radiation in the SME has been studied before, in several different SME parameter regimes. The previous analyses have used a variety of techniques. Some studies have applied Green's functions [11] and the related Feynman diagram techniques directly [12]. Other analyses used coordinate transformations to move the Lorentz violation into the charged matter sector; this can be combined with the observation that when the electromagnetic sector is conventional, Cerenkov radiation occurs exactly if a charge's speed $v$ exceeds 1 [13, 14].

A fully comprehensive picture of Cerenkov radiation in the minimal SME has still not yet been developed, however. This work provides an exact (and highly unexpected) solution to the problem in the timelike Chern-Simons theory - which was among the first renormalizable Lorentz-violating theories to be studied. Rather than working with the Green's functions and dispersion relations for the Lorentz-violating theory, this analysis will work directly with the modified Maxwell's equations. We shall develop a systematic way to find electric and magnetic fields of a uniformly moving charge, perturbatively as a function of the Lorentz violation coefficient and the particle speed. From these fields, the radiation losses may be calculated directly. This technique was first applied in [15] up to $\mathcal{O}\left(v^{2}\right)$, for which only a determination of a single modified field at first order in the Lorentz violation was needed. Here we shall demonstrate how the same kind of calculations may be performed to all orders.

The Lagrange density for the electromagnetic sector of the minimal SME is

$$
\mathcal{L}=-\frac{1}{4} F^{\mu \nu} F_{\mu \nu}-\frac{1}{4} k_{F}^{\mu \nu \rho \sigma} F_{\mu \nu} F_{\rho \sigma}+\frac{1}{2} k_{A F}^{\mu} \epsilon_{\mu \nu \rho \sigma} F^{\nu \rho} A^{\sigma}-j^{\mu} A_{\mu} .
$$

The $k_{F}$ term, which is even under CPT, has been extensively studied. The focus here will be on the $k_{A F}$ term; this is the Chern-Simons term, which is odd under CPT. We shall specifically be interested in a timelike $k_{A F}$ term, which, in an appropriate frame, takes the purely timelike form $k_{A F}^{\mu}=(k, \overrightarrow{0})$. 
There is an obvious difficultly with this theory when we look at the dispersion relations for circularly polarized plane waves. The dispersion relation is $\omega_{ \pm}^{2}=p(p \mp 2 k)$, for modes of helicity \pm 1 . At the longest wavelengths, with $p<|2 k|$, one of the two modes apparently has an imaginary frequency; as we will see, this is related to the fact that the energy is not bounded below. The existences of imaginary frequencies and unboundedly negative energies are, in turn, tied to the existence of runaway solutions, in which the field grows without bound. It is possible to avoid the runaway solutions by using an acausal Green's function [3]. However, there is a cost, lying precisely in the acausality; charged particles may begin to radiate before they actually start to move. Identifying the correct behavior of a theory with a timelike Chern-Simons term thus becomes a tricky problem. Fortunately, however, this problem does not exist when we study the Cerenkov radiation from a charge that moves with a perfectly constant velocity.

To understand the radiation of energy in a modified electromagnetic theory, we must look at the energy-momentum tensor. For an arbitrary $k_{A F}$, this tensor takes the form [3]

$$
\Theta^{\mu \nu}=-F^{\mu \alpha} F_{\alpha}^{\nu}+\frac{1}{4} g^{\mu \nu} F^{\alpha \beta} F_{\alpha \beta}-\frac{1}{2} k_{A F}^{\nu} \epsilon^{\mu \alpha \beta \gamma} F_{\beta \gamma} A_{\alpha} .
$$

For the purely timely $k_{A F}$, the energy density $\left(\Theta^{00}\right)$, momentum density $\left(\Theta^{0 j}\right)$, and energy flux $\left(\Theta^{j 0}\right)$ are

$$
\begin{aligned}
\mathcal{E} & =\frac{1}{2} \vec{E}^{2}+\frac{1}{2} \vec{B}^{2}-k \vec{B} \cdot \vec{A} \\
\overrightarrow{\mathcal{P}} & =\vec{E} \times \vec{B} \\
\vec{S} & =\vec{E} \times \vec{B}-k A_{0} \vec{B}+k \vec{A} \times \vec{E} .
\end{aligned}
$$

The asymmetry of $\Theta^{\mu \nu}$ is a general feature of Lorentz-violating theories. However, other unusual features are specific to the theory with $k_{A F}$. The energy density is not gauge invariant, although the total energy, integrated over all space, is a gauge-invariant quantity. Moreover, as previously noted, $\mathcal{E}$ is not bounded below, and this feature will turn out to plan a crucial role in understanding the absence of Cerenkov radiation.

To study Cerenkov radiation, we will look at the fields produced by a charged particle $q$ moving along the trajectory $\vec{r}(t)=v t \hat{z}$, taken at time $t=0$. A particle emitting Cerenkov radiation would of be subject to recoil; however, we shall neglect any effects of recoil here. (Any radiation that is directly a result of recoil effects is not really Cerenkov radiation, since it is caused by a secondary acceleration.) For a particle in completely steady motion, all the fields it produces must similarly be moving along in the $z$-direction with speed $v$, and this will provide a tremendous simplification of the time derivatives that appear in Maxwell's equations.

We write the electric and magnetic fields in this scenario as series

$$
\vec{E}=\sum_{m=0}^{\infty} \sum_{n=0}^{\infty} \vec{E}^{(m, n)}
$$




$$
\vec{B}=\sum_{m=0}^{\infty} \sum_{n=1}^{\infty} \vec{B}^{(m, n)} .
$$

In each sum, a term with superscripts $(m, n)$ is proportional to $k^{m} v^{n}$. These power seies forms will allow us to pick out the terms that can contribute to our expression for the radiated energy. The usual terms are the $\vec{E}^{(0, n)}$ with $n$ even and $\vec{B}^{(0, n)}$ with $n$ odd.

The only modification of Maxwell's equations is to the Ampere-Maxwell law; the modified version is

$$
\vec{\nabla} \times \vec{B}=\frac{\partial \vec{E}}{\partial t}+2 k \vec{B}+\vec{J}
$$

The other three equations are just as usual. The time derivatives of the fields simplify considerably, since we know that the steady-state field profiles must be moving in the $z$-direction uniformly in time. By considering the steady-state scenario, we avoid having to deal with the question of whether an acausal Green's function in required. A field (either $\vec{E}$ or $\vec{B})$ must have the form $\vec{W}(\vec{r}, t)=\vec{W}(\vec{r}-v \hat{z} t, 0)$, and this means that any time derivative $\frac{\partial}{\partial t}$ acting on $\vec{E}$ or $\vec{B}$ may be replaced with $-v \frac{\partial}{\partial z}$.

The modified fields may thus be generated iteratively, according to

$$
\begin{aligned}
\vec{\nabla} \times \vec{E}^{(m, n)} & =v \frac{\partial \vec{B}^{(m, n-1)}}{\partial z} \\
\vec{\nabla} \cdot \vec{E}^{(m, n)} & =0 \\
\vec{\nabla} \times \vec{B}^{(m, n)} & =-v \frac{\partial \vec{E}^{(m, n-1)}}{\partial z}+2 k \vec{B}^{(m-1, n)} \\
\vec{\nabla} \cdot \vec{E}^{(m, n)} & =0,
\end{aligned}
$$

starting with the Lorentz-invariant fields $\vec{E}^{(0, n)}$ and $\vec{B}^{(0, n)}$. Successively solving these equations provides terms with increasing $m+n$. Note that no terms beyond $m=2$ can contribute to the energy loss; for dimensional reasons, the total power radiated must have mass dimension 2, and there is no other dimensional scale apart from $k$ in the problem.

Understanding the general properties of the $\vec{E}^{(m, n)}$ and $\vec{B}^{(m, n)}$ is crucially important. The standard fields $\vec{E}^{(0, n)}$ and $\vec{B}^{(0, n)}$ involve only even powers of $v$ for the electric terms and odd powers of $v$ for the magnetic terms. Looking at the equations (9-12), it is evident that $\vec{E}$ remains an even function of $v$ and $\vec{B}$ an odd function to all orders. However, there are no similar conditions on the parity of the solutions with respect to $k$.

Geometrically, the $k$-dependent $\vec{E}^{(m, n)}$ and $\vec{B}^{(m, n)}$ terms may take two forms; they may be either azimuthal or toroidal. An azimuthal field is divergenceless and points in the $\hat{\phi}$-direction, and its magnitude is independent of $\phi$; the $\vec{B}^{(0, n)}$ all have this form. A toroidal field is likewise divergenceless, and it points entirely in the $\hat{r}$ - and $\hat{\theta}$-directions; and again, these components are independent of $\phi$. Whichever geometric structure a given term $\vec{W}$ has, its derivative $\partial \vec{W} / \partial z$ will have the same structure. (The $z$-derivative of a divergenceless field remains divergenceless; nor will the derivative introduce any explicit 
dependence on $\phi$; nor does taking the derivative mix the $\{\hat{r}, \hat{\theta}\}$ and $\{\hat{\phi}\}$ spaces of unit vectors.)

A key point is that if the sources for a given term [that is, the fields appearing on the right-hand sides of (9) and (11)] are toroidal, then the terms they generate (on the left) are azimuthal, and vice versa. These are both standard facts. The most elementary examples of these statements are that the magnetic field of a circular loop of current is toroidal, and the magnetic field of a toroid of uniform cross section wound with wire is azimuthal. The general statements can, in fact, be derived simply by taking superpositions of sources with the geometries appearing those two examples. Since the $\vec{B}^{(0, n)}$ (from which all the $k$-dependent terms are ultimately derived) are azimuthal, it follows (by trivial induction) that a field has toroidal geometry whenever $m+n$ is even and azimuthal geometry when $m+n$ is odd. No term has a mixture of the two geometry types.

The solutions of the equations (9-12) may be found by elementary means. The problem is easier when the source terms are toroidal, so that the term they generate is azimuthal. The magnitude of the azimuthal component may be found using a pseudo-Amperean technique. Consider a circle $C$ with radius $\rho$, lying parallel to the $x y$-plane and with its center at $(0,0, z)$. For concreteness, we will look at how $\vec{B}^{(2,1)}$ is generated from the $\vec{B}^{(1,1)}=\frac{k q v}{4 \pi r}(2 \cos \theta \hat{r}-\sin \theta \hat{\theta})$ previously calculated in [15]. We have

$$
\begin{aligned}
\int_{C} d \vec{l} \cdot \vec{B}^{(2,1)}=2 \pi \rho B_{\phi}^{(2,1)} & =\int_{0}^{\rho}\left(2 \pi \rho^{\prime} d \rho^{\prime}\right) \vec{B}^{(1,1)} \cdot \hat{z} \\
& =\frac{k q v}{2} \int_{0}^{\rho} d \rho^{\prime} \frac{\rho^{\prime}}{\sqrt{\rho^{\prime 2}+z^{2}}}\left(1+\frac{z^{2}}{\rho^{\prime 2}+z^{2}}\right) \\
& =\frac{k q v}{2} \frac{\rho^{2}}{\sqrt{\rho^{2}+z^{2}}} \\
\vec{B}^{(2,1)} & =\frac{k q v}{4 \pi} \sin \theta \hat{\phi}
\end{aligned}
$$

This has a singularity at the origin, but so does its source, and so indeed do all the fields.

It will also be critical to understand whether the components of the $\vec{E}^{(m, n)}$ and $\vec{B}^{(m, n)}$ are odd or even functions of $\cos \theta$ (or equivalently of $z$ ). This will be referred to as the $z$-parity. Ultimately, the $z$-parity will be important because if $\vec{S} \cdot \overrightarrow{\hat{r}}$ is an odd function of $\cos \theta$, there will be no net outflow of energy.

In the example calculation just considered, with $\vec{B}^{(1,1)}$ generating $\vec{B}^{(2,1)}$, the source could be written as $\vec{B}^{(1,1)}=f(r)[O(\theta) \hat{r}+E(\theta) \hat{\theta}]$, where $f(r)$ contains the radial dependence, $O(\theta)$ is an odd function of $\cos \theta$ (although, obviously, not an odd function of $\theta$ itself), and $E(\theta)$ is an even function of $\cos \theta$. Then $\vec{B}^{(1,1)} \cdot \hat{z}=f(r)[O(\theta) \cos \theta-E(\theta) \sin \theta]$ is strictly an even function of $\cos \theta$. The flux of this field through a circular loop centered at $(0,0, z)$ is the same as it would be if the loop were centered at $(0,0,-z)$; this makes the azimuthal $\vec{B}^{(2,1)}$ an even function of $\cos \theta$. Obviously, this generalizes to other sources with the same $z$-parity structure; and a source term with the opposite $z$-parity 
structure [proportional to $E(\theta) \hat{r}+O(\theta) \hat{\theta}$ ] will generate an new azimuthal field term with odd z-parity.

In addition to $\vec{B}^{(m, n)}$ serving directly as a source (via the novel term in Maxwell's equations), there are also the source terms in the usual electromagnetic induction expressions, which involve derivatives. The $z$-derivative operator

$$
\frac{\partial}{\partial z}=\cos \theta \frac{\partial}{\partial r}+\frac{\sin ^{2} \theta}{r} \frac{\partial}{\partial \cos \theta}
$$

reverses the $z$-parity of any field it acts on. Continuing with the example of a $\vec{B}^{(1,1)}$ source, we have

$$
\frac{\partial \vec{B}^{(1,1)}}{\partial z}=-\frac{k q v}{4 \pi r^{2}}\left(3 \cos ^{2} \theta-1\right) \hat{r}
$$

which does indeed have the $E(\theta) \hat{r}+O(\theta) \hat{\theta}$ form [albeit with $O(\theta)=0$ ].

The result of the preceding paragraphs' analysis is that with a toroidal $\vec{B}^{(m, n)}$ - having odd $z$-parity in the $r$-component and even $z$-parity in the $\theta$-component-the new field terms generated through (9) and (11) will be an azimuthal $\vec{B}^{(m+1, n)}$ with even $z$-parity and an azimuthal $\vec{E}^{(m, n+1)}$ with odd $z$-parity. For a toroidal $\vec{E}^{(m, n)}$ with the opposite $z$-parities (odd for the $r$-component and even for the $\theta$-component) the sole new term generated will be an azimuthal $\vec{B}^{(m, n+1)}$, again with even $z$-parity.

The determinations of the new toroidal field terms generated by an azimuthal source term is slightly tricky. There is no pseudo-Amperean technique for simply reducing the solution of the problem to a definite integral. However, the problem can be reduced to that of solving a single ordinary differential equation. The key to this reduction is noticing that the dependence of any field on $r$ is completely determined by its dependence on $k$. For dimensional reasons alone, $k$ and $r$ will always appear in a single combined factor of $\frac{k^{m}}{r^{2-m}}$. Then we may take, for example, $\vec{E}^{(m, n)}$ to be $\vec{E}^{(m, n)}=\frac{1}{r^{2-m}}[X(\theta) \hat{r}+Y(\theta) \hat{\theta}]$, so that the curl and divergence equations reduce to

$$
\begin{aligned}
\vec{\nabla} \times \vec{E}^{(m, n)} & =\frac{1}{r^{3-m}}\left[-X^{\prime}(\theta)+(m-1) Y(\theta)\right] \hat{\phi}=v \frac{\partial B_{\phi}^{(m, n-1)}}{\partial z} \\
\vec{\nabla} \cdot \vec{E}^{(m, n)} & =\frac{1}{r^{3-m}}\left[m X(\theta)+\cot \theta Y(\theta)+Y^{\prime}(\theta)\right]=0 .
\end{aligned}
$$

The prime in $X^{\prime}$ denotes the derivative with respect to the argument $\theta$. Note that if $X$ and $Y$ are functions of well-defined $z$-parity, (20) implies that the $z$-parities for the two component functions $X$ and $Y$ are opposite.

For $m \neq 1$, (19) may be used to eliminate $Y$. The resulting second-order differential equation is

$$
X^{\prime \prime}+\cot \theta X^{\prime}+m(m-1) X=v r^{3-m}\left[\cot \theta \frac{\partial B_{\phi}^{(m, n-1)}}{\partial z}-\sin \theta \frac{\partial}{\partial \cos \theta} \frac{\partial B_{\phi}^{(m, n-1)}}{\partial z}\right],
$$




\begin{tabular}{|c|c|c|c|}
\hline & & $z$-Parity & \\
Field Term & $\hat{r}$ & $\hat{\theta}$ & $\hat{\phi}$ \\
\hline Toroidal $\vec{E}$ & + & - & $\emptyset$ \\
Azimuthal $\vec{E}$ & $\emptyset$ & $\emptyset$ & - \\
Toroidal $\vec{B}$ & - & + & $\emptyset$ \\
Azimuthal $\vec{B}$ & $\emptyset$ & $\emptyset$ & + \\
\hline
\end{tabular}

Table 1: $z$-parity values for the four different types of field terms: + and - denote even and odd parity, respectively; $\emptyset$ denotes that the corresponding term is zero for fields of the indicated type.

using $\frac{\partial}{\partial \theta}=-\sin \theta \frac{\partial}{\partial \cos \theta}$. There is a particular solution of this equation that is proportional to $k^{m} v^{n}$ and has no singularities in $\theta$. In all cases that we have specifically investigated, this solution could be found using the Ansatz that $X(\theta)$ be a polynomial in $\cos \theta$. Then $Y$ follows from (19). For example, the solution for $\vec{E}^{(2,2)}$ [sourced by a $\vec{B}^{(2,1)}$ ] is

$$
\vec{E}^{(2,2)}=-\frac{k^{2} q v^{2}}{4 \pi}\left[\left(\frac{3}{2} \cos ^{2} \theta-\frac{1}{2}\right) \hat{r}-\sin \theta \cos \theta \hat{\theta}\right] .
$$

However, the key general point [evident from inspection of (21)] is that the nonsingular particular solution $X$ has the same $z$-parity as $B_{\phi}^{(m, n-1)}$. As noted, $Y$ then has the opposite $z$-parity. (For the previously excluded $m=1$ case, the results concerning the $z$-parity of $X$ and $Y$ are the same. However, the calculations at that order are simpler; only solutions of first order linear differential equations are required.)

Collecting these results, we have that an azimuthal $\vec{B}^{(m, n)}$ with even $z$-parity produces a toroidal $\vec{B}^{(m+1, n)}$ with odd $z$-parity in the $\hat{r}$ component and even $z$-parity in the $\hat{\theta}$ component, as well as a toroidal $\vec{E}^{(m, n+1)}$ with even $z$-parity along $\hat{r}$ and odd for $\hat{\theta}$. An azimuthal $\vec{E}^{(m, n)}$ with odd $z$-parity produces a toroidal $\vec{B}^{(m, n+1)}$ with odd $z$-parity in $\hat{r}$ and even $z$-parity in $\hat{\theta}$.

Since the $k$-independent fields $\vec{E}^{(0, n)}$ and $\vec{B}^{(0, n)}$ have well-defined $z$-parities, the higher order field terms must as well. The z-parity values for the possible fields, as determined from the rules we have derived, are listed in table 1. Knowing these symmetry properties, it is possible to demonstrate that there is no outgoing energy flux at spatial infinity, up to any order in $v$.

The energy flux $\vec{S}$ is composed of the various fields and potentials. A net outward power loss is $P=R^{2} \int d \Omega \vec{S} \cdot \hat{r}$, with the integral taken as $R \rightarrow \infty$. Note that while $\mathcal{E}$ and $\vec{S}$ are not gauge invariant, the integral of $\vec{S} \cdot \hat{r}$ over the sphere at infinity is a gauge invariant quantity. If the integrand $\vec{S} \cdot \hat{r}$ itself has odd $z$-parity, then integral automatically vanishes.

A number of different components of $\vec{E}$ and $\vec{B}$ may contribute to $\vec{S} \cdot \hat{r}$. The conventional 
term $(\vec{E} \times \vec{B}) \cdot \hat{r}$-also the only term appearing in the momentum outflow-receives contributions proportional to $E_{\theta} B_{\phi}$ and $E_{\phi} B_{\theta}$; both of these combinations have odd $z$ parity. The remaining two terms involve the potentials $A_{0}$ and $\vec{A}$. Since the corresponding terms in $\vec{S}$ depend on $k$ explicitly, and the final power $P$ must be proportional to $k^{2}$, any contributing terms must involve either $\vec{E}^{(1, n)}$ or $\vec{B}^{(1, n)}$, as the $\vec{E}^{(0, n)} \propto \hat{r}$ and $\vec{B}^{(0, n)} \propto \hat{\phi}$ conventional terms obviously point in the wrong directions to contribute. The potential terms needed are thus only the conventional

$$
\begin{aligned}
A_{0} & =\frac{q}{4 \pi r \sqrt{1-v^{2} \sin ^{2} \theta}} \\
\vec{A} & =\frac{q v \hat{z}}{4 \pi r \sqrt{1-v^{2} \sin ^{2} \theta}} .
\end{aligned}
$$

This $A_{0}$ has even $z$-parity, and the components of $\vec{A}$ have the same $z$-parities as a the components of a toroidal $\vec{B}$. The combinations involving these potentials that can contribute to $\vec{S} \cdot \hat{r}$ are thus proportional to $A_{0} B_{r}, A_{\theta} E_{\phi}$, and $A_{\phi} E_{\theta}$. Again, all of these bilinears have odd $z$-parity.

Strictly speaking, the condition that $P$ be proportional to $k^{2}$ only applies if $P$ is finite. This is not an idle concern; in the CPT-even theory with $k_{F}$, the rate of Cerenkov losses for a charge moving with constant speed is in fact infinite, and only recoil corrections or additional higher-dimensional operators make the actual power emitted finite. However, unlike in the $k_{F}$ theory, in the Chern-Simons theory there is a hard upper limit on the energies of the emitted photons, above which radiation is kinematically disallowed. Moreover, it is actually a straightforward matter to generalize our analysis of the $z$-parity to the $k$-dependent potential terms. This is particularly transparent for the vector potential, for which the source relations in the Coulomb gauge are

$$
\begin{aligned}
\vec{\nabla} \times \vec{A}^{(m, n)} & =\vec{B}^{(m, n)} \\
\vec{\nabla} \cdot \vec{A}^{(m, n)} & =0,
\end{aligned}
$$

very similar to (11 12). So the $\vec{A}$ terms have the same $z$-parities as $\vec{B}$ terms of the same geometric types. The structure of $A_{0}$ is slightly trickier; the lone source equation for the scalar potential is

$$
\vec{\nabla} A_{0}^{(m, n)}=-\vec{E}^{(m, n)}+v \frac{\partial \vec{A}^{(m, n-1)}}{\partial z} .
$$

The solutions $A_{0}^{(m, n)}$ (which only exist for $m+n$ even) have even $z$-parity-leading to $\vec{\nabla} A_{0}^{(m, n)}$ with even $z$-parity in the $\hat{r}$-component and odd $z$-parity in $\hat{\theta}$.

Since all possible contributions to $\vec{S} \cdot \hat{r}$ have odd $z$-parity, the power loss from the moving charge is identically $P=0$. While $\vec{S}$ itself does not vanish at spatial infinity, $\vec{S}$ on its own is not a gauge invariant quantity and has no physical meaning. Only the integral can be measured. However, even taking this into account, $P=0$ is a puzzling result. The 
previous analysis [15] found that $P=0$ up to $\mathcal{O}\left(v^{2}\right)$; however, a phase space estimate of the power emitted at $\mathcal{O}\left(v^{3}\right)$ was nonzero. Yet that estimate was based on an analysis only of the modes with $p>|2 k|$. For any $v>0$, there are $p>|2 k|$ modes of the electromagnetic field with phase speeds slower than $v$, and on general principles, it appears there should be energy radiated into these modes. However, the phase space analysis is inapplicable for the $p<|2 k|$ modes, which do not have well-defined real frequencies. The behavior of these long-wavelength modes is actually key to understanding why the total power emitted vanishes. Since the energy density in the Chern-Simons theory is unbounded below, these modes of the field actually carry away negative energy that precisely cancels the positive energies borne away from the moving charge by the shorter-wavelength modes. Remarkably, in this case, the lack of a lower bound on the energy actually makes the theory better behaved than naive expectations would indicate; for a charged particle moving with an arbitrary velocity $v$ in the timelike Chern-Simons theory, there is no energy loss to vacuum Cerenkov radiation - just like in conventional electromagnetism.

We have established the primary result, that there are no Cerenkov energy losses in this model. However, this is still considerably more to delve into. While no energy is lost by a charge in uniform motion, propagating electromagnetic modes are excited. The phase space estimates of emission spectrum outlined in [14, 15] ought to tell us something about the spectrum, even if they certainly do not tell the whole story. A detailed, mode-by-mode understanding of the radiation modes would be interesting, although it is clearly a complicated problem - not least because the gauge dependence of $\mathcal{E}$ may make it impossible to uniquely identify the energy carried by a particular mode. Alternatively, it might be possible to obtain closed form expressions for $\vec{E}$ and $\vec{B}$ fields. Many of the terms we have evaluated explicitly have components proportional either to $P_{\ell}(\cos \theta)$ or $P_{\ell}(\cos \theta) \sin \theta$, in terms of Legendre polynomials. It might be possible, using functional identities, to resum the power series to get a complete solution for the fields. Either one of these avenues would be quite interesting, but the present work has demonstrated the most important result, that the Cerenkov power loss rate in the timelike Chern-Simons theory is $P=0$.

\section{References}

[1] D. Colladay, V. A. Kostelecký, Phys. Rev. D 55, 6760 (1997).

[2] D. Colladay, V. A. Kostelecký, Phys. Rev. D 58, 116002 (1998).

[3] S. M. Carroll, G. B. Field, R. Jackiw, Phys. Rev. D 41, 1231 (1990).

[4] V. A. Kostelecký, M. Mewes, Phys. Rev. Lett. 99, 011601 (2007).

[5] S. Coleman, S. L. Glashow, Phys. Rev. D 59, 116008 (1999). 
[6] R. Jackiw, V. A. Kostelecký, Phys. Rev. Lett. 82, 3572 (1999).

[7] M. Pérez-Victoria, Phys. Rev. Lett. 83, 2518 (1999).

[8] J. M. Chung, Phys. Lett. B 461, 138 (1999).

[9] A. A. Andrianov, P. Giacconi, R. Soldati, JHEP 02, 030 (2002).

[10] B. Altschul, Phys. Rev. D 69, 125009 (2004).

[11] R. Lehnert, R. Potting, Phys. Rev. D 70, 125010 (2004); erratum ibid. 70, 129906 (2004).

[12] C. Kaufhold, F. R. Klinkhamer, Nucl. Phys. B 734, 1 (2006).

[13] B. Altschul, Phys. Rev. Lett. 98, 041603 (2007).

[14] B. Altschul, Phys. Rev. D 75, 105003 (2007).

[15] B. Altschul, Phys. Rev. D 90, 021701 (R) (2014). 